\title{
Particles and lungs - where chemical engineering meets medicine
}

\author{
Sosnowski Tomasz R. \\ Faculty of Chemical and Process Engineering, Warsaw University of Technology, POLAND, Warsaw, \\ Waryńskiego 1, E-mail: tomasz.sosnowski@pw.edu.pl
}

\begin{abstract}
Paper draws attention to chemical engineering applications in the problems of drug delivery by inhalation, i.e. in atomization of liquids, preparation of functional powders and their aerosolization, aerodynamic design of inhaling devices, and finally-flow and mass transfer processes in the layers of lung fluids (bronchial mucus and pulmonary surfactant).
\end{abstract}

Keywords - aerosol, generation, inhalation, CFD, surface effects, lung fluids.

\section{Introduction}

The XXI-th century paradigms of chemical engineering emphasize the importance of intensification of the momentum, heat, and mass transfer processes by employing novel approaches which may be based, e.g. on mimicking the phenomena found in the living world ("bio-inspiration"). For instance, microfluidic systems which have been demonstrated to be very effective in conducting mass transfer processes with a high selectivity [1] are inspired by living microsystems which are characterized by extremely high surface-to-area ratio and laminar flowcontrolled mass exchange (e.g. oxygen transport from blood capillaries to the tissues, gasexchange across air/blood barrier in the pulmonary alveoli). This is why the bioengineering becomes very important in the modern chemical and process engineering/technology. Such definition of "bioengineering" has much broader meaning than "biotechnology" or "bioprocess engineering", and it often relates also to the application of the engineering-based analysis of fluid flow and mass transfer phenomena in the close relation to medicine.

The current paper is focused on the highlighting of the problem of particle inhalation and their interactions with the respiratory system, and analyzing these processes by using the phenomenological approach and scientific tools of chemical engineering.

\section{Characteristics of inhaled aerosols and definition of engineering challenges}

Inhaled particles constitute a dispersed colloidal system of polydisperse micro- or nanometer-size objects suspended in the air ("aero" + "sol"). While inhaled, they travel with the air through the complex system of air ducts of the bronchial tree where they can be gradually removed (captured) due to the action of several deposition mechanism, which are essentially identical to those met in the industrial gas cleaning devices (dust separators). Regional deposition in the respiratory tract depends on many factors, including particle size, shape and density, but also the local and temporal air velocity, and the local geometry of the air ducts of the breathing organ (bending, division, narrowing, etc), Figure 1.

When inhaled particles are potentially toxic - such as aerosols generated by combustion or other industrial processes - their local deposition in different parts of the respiratory tract is responsible for many health effects, including airway inflammation, asthma, COPD, fibrosis, and lung cancer. It is commonly agreed that only particles smaller than $10 \mu \mu \mathrm{m}$ are inhalable, i.e. can pass the mouth/throat region ("oropharynx"). Penetration to the deeper regions of the bronchial tree ("lung periphery") requires particles or droplets smaller than $5 \mu \mathrm{m}$ which are often called "fine particles". In the environmental toxicology it has been established that, so called PM2.5 (particulate matter with size below $2.5 \mu \mathrm{m}$ ) form the most harmful aerosol fraction. 


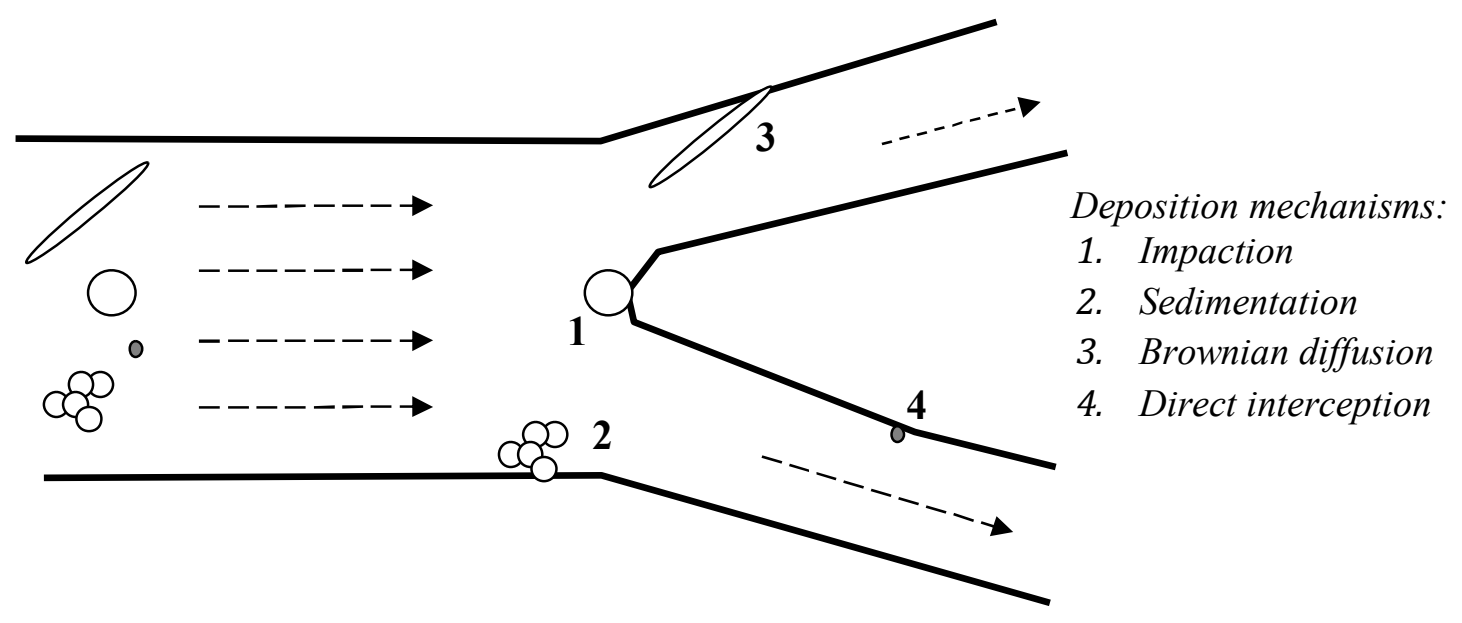

Fig. 1. Schematic deposition of aerosol particles with different shapes and sizes in a bronchial bifurcation.

On the other hand, aerosol particles or droplets may be used also as carriers of medicines (drugs) administered to the respiratory system. They can be used to treat pulmonary diseases locally or to deliver drugs to the whole body via the air/blood barrier [2, 3]. For these therapeutic aerosols we need to predict the spatial distribution of deposition to correctly design the most beneficial drug delivery system (Figure 2).

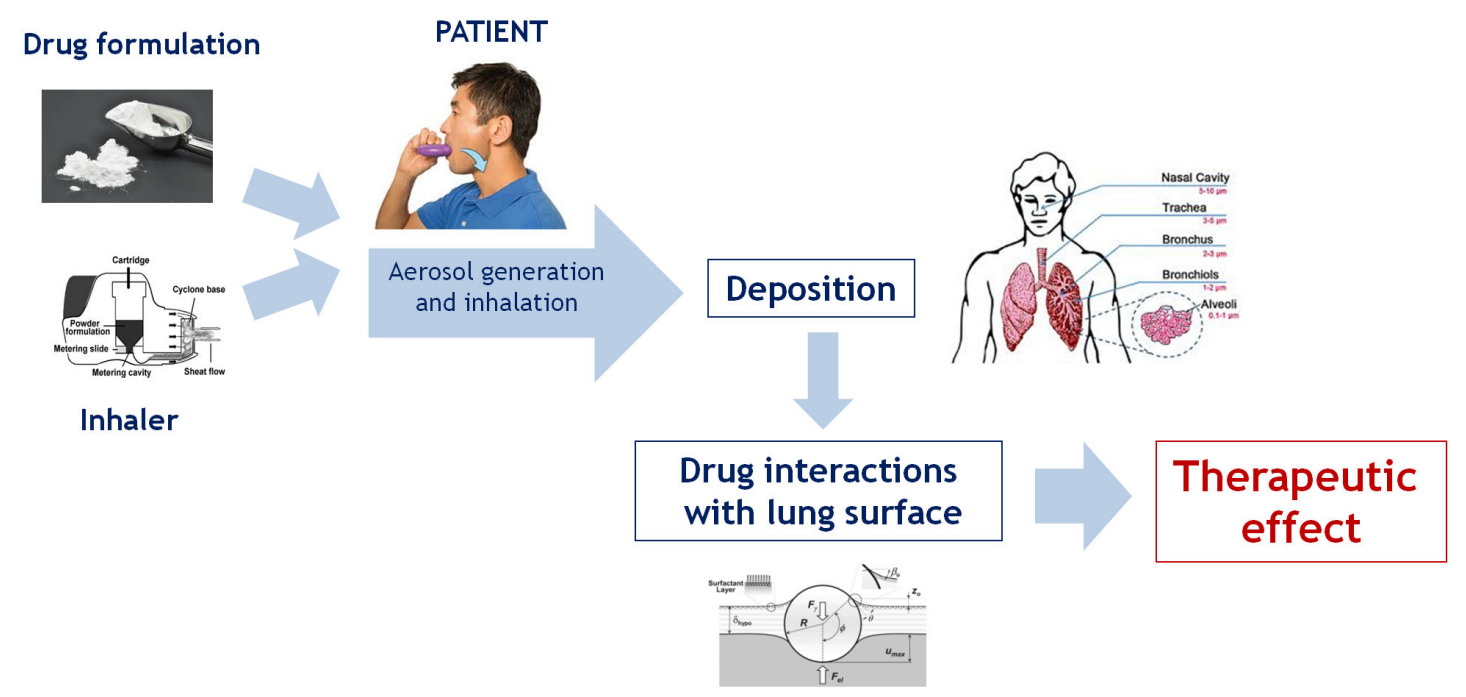

Fig. 2. A scheme of inhalation as a route of drug delivery

As seen in Fig. 2, the essential engineering challenge of drug delivery by inhalation is designing the proper aerosol generating system composed of the carrier (drug formulation) and the aerosolization device (inhaler) capable of producing the inhalable aerosol dose with a desired particle size just before the moment of drug administration. 


\section{Aerosol drug carriers and the dispersing devices (inhalers)}

Inhaled drugs may be carried inside aerosol composed of liquid droplets or solid particles. Consequently, such aerosols are produced in inhalers either by the process of liquid atomization (spraying, known as "nebulization of drugs") or dispersion of powders. Each type of inhaler requires careful design to assure reproducibility of the quality (i.e. particle size distribution) and the quantity (i.e. dose) of emitted drug, ease of use, durability and a reasonable price.

Nebulizers which spray liquid medicines (originally being a solution or suspension), utilize compressed air, ultrasonics or vibrating mesh technology to produce mist of droplets within the micrometric size range. The quality of aerosol mist often depends on the physicochemical properties of nebulized drugs, mainly, the viscosity, surface tension and ionic strength [4-6]. The main advantage of nebulizers is that they are used during normal (tidal) breathing, without special inhalation maneuvers, which make them suitable also for toddlers or people with disabilities. Recently some novel aspects of using these devices have been identified and discussed [7].

Dry powder inhalers (DPIs) are a broad class of medical devices used to disperse powders. They are portable but usually require special inhalation maneuver to assure good generation of aerosol and efficient drug delivery to the lungs. The main problem with DPIs is that they operate properly only if a certain inspiration effort is achieved for producing the inhalable dose, however sometimes such effort cannot be obtained by users (patients). As a consequence the drug may be not delivered as prescribed (aerosol emission is too small, particles are aggregated and too big in size). Various DPIs have different internal aerodynamic resistance which make them even more difficult to use reproducibly by different patients [8]. It is therefore essential to look for the methods to improve the performance of DPIs. One strategy is to optimize the properties of powder to make it easier to disperse. "Particle engineering" is the most suitable approach to do so, and it can use, e.g., the optimized spray drying process to obtain powders with controlled properties regarding their morphology, stability and aerosolization properties [9-12]. The spray drying technique also allows to produce particles with the ordered structure [13] and with certain functionality after deposition on the lung surface [14-16]. An example of a structurized microparticle obtained in our laboratory via controlled spray drying is shown in Fig. 3.

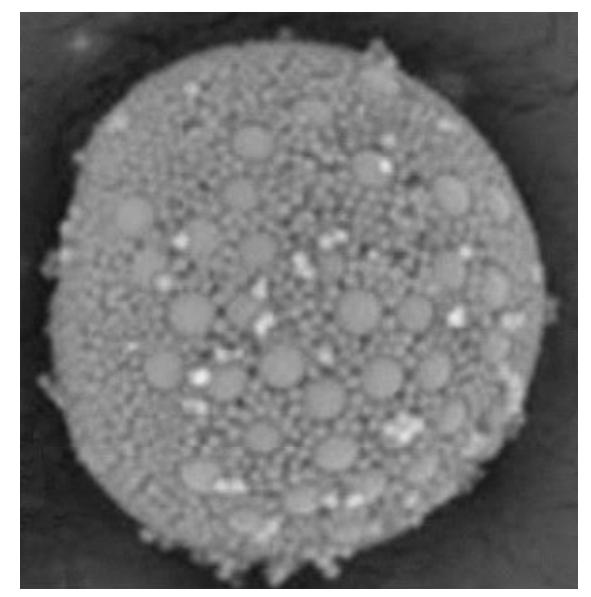

Fig. 3. An example of structurized particle aggregate (size $\sim 10 \mu \mathrm{m}$ ) obtained by controlled distribution of primary spray drying of a colloid with bimodal particles $(250 \mathrm{~nm}$ and $1 \mu \mathrm{m})$. 
It should be pointed out that some novel inhalation drugs are multicomponent i.e. they form a mixture of two or more active compounds delivered during single inhalation, while the dose of each compound is significantly different (e.g., as 10:1). In such cases the problem of the reproducible delivery of each compound in one dose of inhaled aerosol particles with the desired size distribution becomes much more complex and requires a more thorough analysis [17].

Another strategy to increase the efficiency of inhalation with DPIs is the optimization of aerosol dispersing device (inhaler) to obtain the efficient use of airflow energy (provided by an inhaling patient) for the fluidization and deaggregation of powder particles [18, 19]. CFD analysis and experiments on powders dispersion can be very useful in designing new DPI designs, Fig. 4.
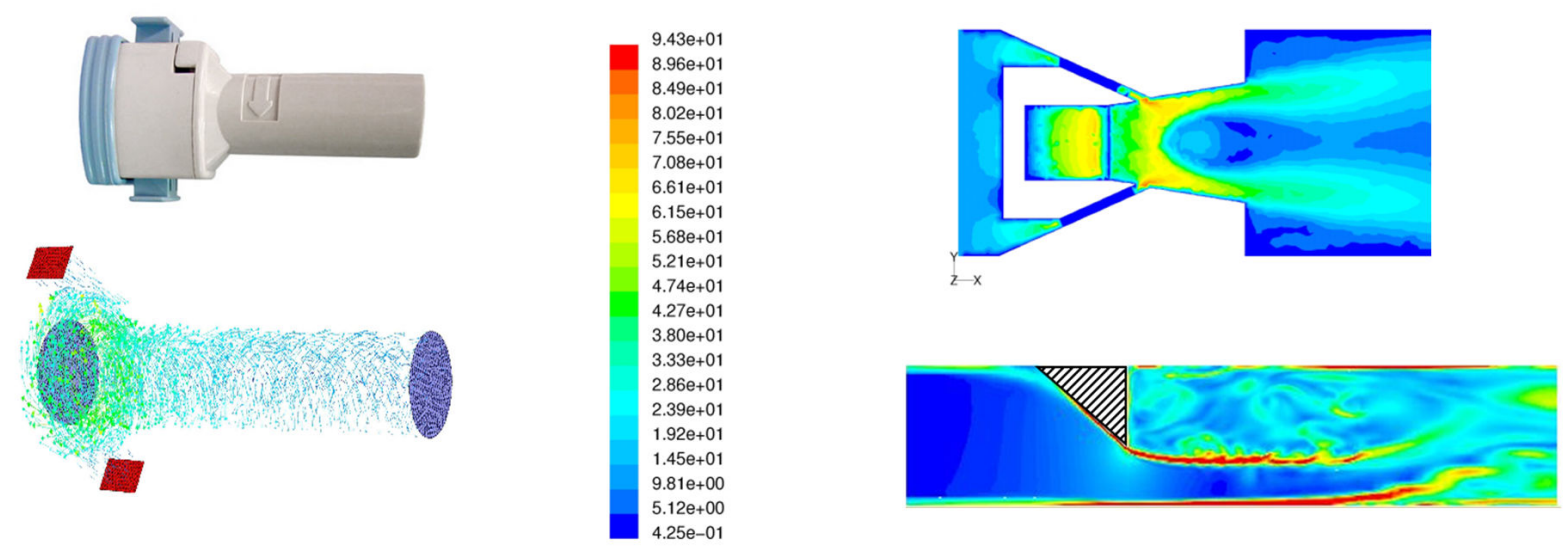

Fig. 4. Examples of use of computational fluid dynamics (CFD) in flow analysis and DPIs design.

\section{Interactions of deposited particles with the lung surface}

According to Fig. 2, the important step in achieving therapeutic effect is related to the interactions of inhaled particles with the lung tissue. This process depends on the interfacial processes which occur when particles are deposited on the internal surface of the respiratory tract. The lung fluids: bronchial mucus and pulmonary surfactant are natural barriers which protect the organism against harmful action of inhaled contaminants. It can be shown by rheological and surface tensiometric measurements done in vitro that inhaled micro- and nanoparticles influence the physicochemical properties of both fluids, and that these effects are dose and material dependent [16 and references therein]. Changes in the dynamic surface tension of the pulmonary surfactant have effects on the mass transfer rate in the alveolar region of the lungs, and this may lead to serious health problems [20-22]. This conclusion is important also for inhaled therapeutics which should be designed in a way to avoid undesired physicochemical changes in the pulmonary surfactant properties $[15,23]$. The problem has been recently raised also in relation to nebulized drugs which often contain surface-active compounds as preservatives and stabilizers of the liquid formulations [24]. 


\section{Conclusions}

This paper outlines selected problems of aerosol inhalation viewed from the chemical engineer's perspective. The discussed problem requires incorporation of several aspects of contemporary chemical engineering, such as the physical chemistry and dynamics of colloidal systems, process intensification, micro-/nanotechnology, and bioengineering, and also often it requires the application of modern research tools (CFD modeling, advanced experimental techniques). It has been demonstrated that such approach can be very effective in a better understanding of interactions of inhaled aerosols with the organism, which is important both in toxicology and therapeutic (medical) applications.

\section{Acknowledgment}

Work supported by NCN project No. 2018/29/B/ST8/00273.

\section{References}

[1] A.J. deMello, "Control and detection of chemical reactions in microfluidic systems", Nature 442, 394-402, 2006.

[2] I. Gonda, "Systemic delivery of drugs to humans via inhalation", J. Aerosol Med. 19, 4753, 1996.

[3] T.R. Sosnowski, „Selected engineering and physicochemical aspects of systemic drug delivery by inhalation”, Curr. Pharm. Des. 22, 2453-2462, 2016.

[4] O.N.M. McCallion, K.M. Taylor, M. Thomas, A.J. Taylor, "Nebulization of fluids of different physicochemical properties with air-jet and ultrasonic nebulizers", Pharm. Res. 12, 1682-1688, 1995.

[5] L. Broniarz-Press, T.R. Sosnowski, M. Matuszak, M. Ochowiak, K. Jabłczyńska, ”The effect of shear and extensional viscosities on atomization of Newtonian and nonNewtonian fluids in ultrasonic inhaler", Int. J. Pharmaceutics 485, 41-49, 2015.

[6] M. Beck-Broichsitter, "Aerosol production by vibrating membrane technology: analysis of the electrolyte effect on generated droplet size and nebulizer output rate", J. Pharm. Sci. 106, 2168-2172, 2017.

[7] T.R. Sosnowski, "Inhaled steroids delivered by mesh nebulizers - what should we know?" Terapia 3s/2019, 1-6, 2019 (in Polish)

[8] M. Pirożyński, T.R. Sosnowski, "Inhalation devices: from basic science to practical use, innovative vs generic products”, Expert Opin. Drug Del. 13, 1559-1571, 2016.

[9] K. Kramek-Romanowska, M. Odziomek, T.R. Sosnowski, L. Gradoń, „Effects of process variables on the properties of spray-dried mannitol and mannitol/disodium cromoglycate powders suitable for drug delivery by inhalation”. Ind. Eng. Chem. Res., 50, 13922-13931, 2011.

[10] M. Odziomek, T.R. Sosnowski, L. Gradon, "Conception, preparation and properties of functional carrier particles for pulmonary drug delivery", Int J. Pharmaceutics 433, 51-59, 2012.

[11] L. Gradoń, T.R. Sosnowski, „Formation of particles for dry powder inhalers”, Adv. Powder Technol. 25, 43-55, 2014. 
[12] K. Jabłczyńska, M. Janczewska, A. Kulikowska, T.R. Sosnowski, „Preparation and characterization of biocompatible polymer particles as potential nanocarriers for inhalation therapy", Int J. Polymer Sci. 2015, article ID: 763020 (8 pages), 2015.

[13] K. Jabłczyńska, J.M. Gac, T.R. Sosnowski, "Self-organization of colloidal particles during drying of a droplet: modeling and experimental study", Adv. Powder Technol. 29, 35423551, 2018.

[14] M. Odziomek, T.R. Sosnowski, L. Gradoń, „The influence of Functional Carrier Particles (FCPs) on the molecular transport rate through the reconstructed bronchial mucus - in vitro studies", Transport in Porous Media 106, 439-454, 2015.

[15] K. Kramek-Romanowska, M. Odziomek, T.R. Sosnowski, „Dynamic tensiometry studies on interactions of novel therapeutic inhalable powders with model pulmonary surfactant at the air-water interface", Colloids Surfaces A: Physicochem. Eng. Aspects 480, 149-158, 2015.

[16] T.R. Sosnowski, "Particles on the lung surface - physicochemical and hydrodynamic effects", Curr. Opin. Colloid Interface Sci. 36, 1-9, 2018.

[17] A. Hejduk, A. Urbańska, A. Osiński, P. Łukaszewicz, M. Domański, T.R. Sosnowski, „Technical challenges in obtaining an optimized powder/DPI combination for inhalation delivery of a bi-component generic drug”, J. Drug Deliv. Sci. Technol. 44, 406-414, 2018.

[18] J. Gac, T.R. Sosnowski, L. Gradoń, „Turbulent flow energy for aerosolization of powder particles", J. Aerosol Sci. 39, 113-126, 2008.

[19] T.R. Sosnowski, K. Giżyńska, Ł. Żywczyk, „Fluidization and break-up of powder particle aggregates during constant and pulsating flow in converging nozzles", Colloids Surfaces A: Physicochem. Eng. Aspects 441, 905- 911, 2014

[20] T.R. Sosnowski, L. Gradoń, M. Skoczek, H. Droździel, „Experimental evaluation of the importance of the pulmonary surfactant for oxygen transfer rate in human lungs", Int. J. Occup. Safety Ergon. 4, 391-409, 1998.

[21] T.R. Sosnowski, A. Podgórski, L. Gradoń, „Influence of insoluble aerosol deposits on the surface activity of the pulmonary surfactant: a possible mechanism of alveolar clearance retardation?" Aerosol Sci. Techn. 32, 52-60, 2000.

[22] D. Kondej, T.R. Sosnowski, „Effect of clay nanoparticles on model lung surfactant: a potential marker of hazard from nanoaerosol inhalation", Environ. Sci. Pollut. Res. 23, 4660-4669, 2016.

[23] T.R. Sosnowski, L. Gradoń, „Modification of inhalable powders by pulmonary surfactant components adsorbed on droplets during spray-drying process", Colloids Surfaces A: Physicochem. Eng. Aspects 365, 56-61, 2010.

[24] K. Dobrowolska, T.R. Sosnowski, „Deposition and interaction of inhalation drugs with the lung surface - model studies", Inż. Aparat Chem. 57(3), 53-54, 2018 (in Polish) 